No. 2

\title{
MONOTROPIC CHARACTER OF POLYMORPHISM IN PENTACHLOROPYRIDINE CRYSTAL STUDIED BY CALORIMETRIC AND SPECTROSCOPIC METHODS
}

\author{
G. WóJCIK \\ Institute of Physical and Theoretical Chemistry, Technical University of Wrocław \\ Wyb. Wyspianskiego 27, 50-370 Wrocław, Poland \\ J. Giermańska-Kahn \\ CNRS, Centre de Recherche Paul Pascal, 33-600 Pessac, France \\ Y. MARqueton \\ Group of Condensed Matter and Materials, University of Rennes I \\ 35-042 Rennes, France \\ AND M. FOULON
}

Laboratory of Dynamics and Structure of Molecular Materials, University of Lille I 59-655 Villeneuve d'Ascq, France

(Received March 13, 1995; in final form May 25, 1995)

Dedicated to Professors Krzysztof Pigon, Józef W. Rohleder and Zdzisław Ruziewicz on the occasion of their 70th birthdays*

Pentachloropyridine crystals in two polymorphic modifications: tetragonal and monoclinic were studied by differential scanning calorimetry and polarized IR and Raman spectroscopy in 20 to $200 \mathrm{~cm}^{-1}$ region and at different temperatures from melting point down to the liquid nitrogen temperature. The $4 \mathrm{~K}$ difference in melting points of both forms indicates the occurrence of monotropy. The spectra were discussed in terms of positional disorder in the studied crystals which often happen to be mixtures of the monoclinic crystal and the L- and D-forms of the tetragonal one. The monotropic transition of the metastable tetragonal crystal into the stable monoclinic one was observed in the Raman spectra and under the polarizing microscope at temperatures ranging from 170 to $185 \mathrm{~K}$ and as an anomaly on the differential scanning calorymetry diagram at $193 \mathrm{~K}$.

PACS numbers: $64.70 . \mathrm{Kb}, 78.30 .-\mathrm{j}$

* Submitted on invitation of the Institute of Physical and Theoretical Chemistry, Technical University of Wrocław, Wrocław, Poland. 


\section{Introduction}

The polymorphism of pentachloropyridine (PCP) was described by Russel and Scott [1] who found the crystal structures of two forms and revealed the optical activity of the tetragonal crystal. Both forms: the low-temperature monoclinic and the high-temperature tetragonal ones have very similar crystal structures consisting of molecular layers parallel to (001) plane as it is shown in Fig. 1. Their
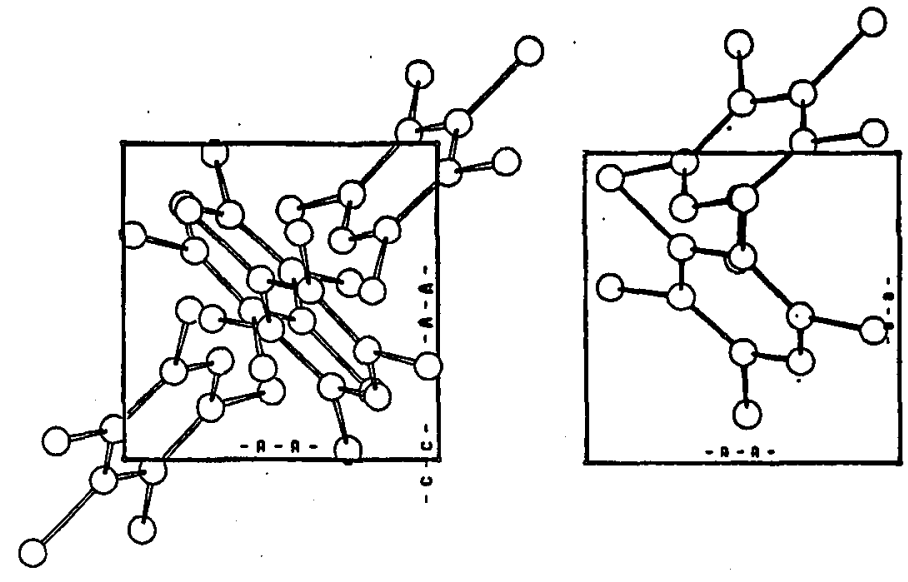

Fig. 1. The (001) projection of the tetragonal (left) and monoclinic (right) unit cell of PCP.

lattice parameters are almost the same except the $c$ one which is doubled in the tetragonal structure. They are presented in Table I. In the tetragonal crystal the molecules are chirally arranged along the fourfold $c$ axis forming a helix. Such an arrangement is accounted for the optical activity of the tetragonal crystals. They may occur in right hand- and left hand forms or as racemate mixture.

\section{TABLE I}

The crystallographic parameters of the tetragonal and monoclinic forms of PCP, according to [1].

\begin{tabular}{l|l}
\hline \hline Tetragonal & Monoclinic \\
\hline$P 4_{1} 2_{1} 2$ & $P 2_{1}$ \\
$a=5.297 \AA$ & $a=5.353 \AA$ \\
& $b=5.239 \AA$ \\
$c=29.785 \AA$ & $c=15.100 \AA$ \\
& $\beta=100.12^{\circ}$ \\
$Z=4$ & $Z=2$
\end{tabular}


According to the X-ray diffraction data [1] the monoclinic crystals are disordered so that $30 \%$ of the molecules are rotated in their own plane by $180^{\circ}$ around the ring centre. The energy calculations with the atom-atom potential [2] excluded however the dynamical character of reorientational disorder. It seems that the disorder is the result of easy twinning of the monoclinic crystals. Their unit cells contain half of the helix of the tetragonal unit cell. The twinned monoclinic crystals with the (001) composition plane imitate well the tetragonal ones and can even intergrow with them. No evidence of any phase transition was found [1].

\section{Experimental}

PCP from "Fluka" was purified by recrystallization from $\mathrm{CCl}_{4}$ and repeated sublimation under vacuum. The single crystal specimens were obtained directly from the sublimation tube or by slow evaporation of the $\mathrm{CCl}_{4}$ solution. Many of them grew in form of pyramids and they had the tetragonal symmetry. Others which had more rectangular shape were monoclinic. The polymorphs were distinguished and oriented by X-rays or optically under the polarizing microscope owing to their distinctly different optical properties, namely, the different interference figures in the conoscopic light and under crossed polarizers [3].

The single crystal specimens of both polymorphs were studied by differential scanning calorimetry (DSC), the IR and Raman spectroscopy in the lattice vibrations region and under the polarizing microscope. The DSC diagrams were measured on the Perkin-Elmer DSC7 apparatus which was calibrated on the melting temperature of indium for temperatures above ambient and on the temperature of the phase transition of adamantane $(208.6 \mathrm{~K})$ for temperatures below ambient. We used samples weighing 10-30 $\mathrm{mg}$ and different rates of heating and cooling cycles. The polarized far IR spectra were measured on a Fourier transform spectrometer coupled with a computer. The resolution was about $1.5 \mathrm{~cm}^{-1}$. The Raman spectra in the $20-200 \mathrm{~cm}^{-1}$ region were measured in all polarizations and at different temperatures from ambient to the liquid nitrogen one with a Coderg LRDM Spectrometer using $647.1 \mathrm{~nm}$ beam from a krypton laser for excitations.

The interference figures under crossed polarizers were observed in the $(a, a)$ and $(a, b)$ planes of single crystal specimens at different temperatures below the ambient one.

\section{The DSC results}

The DSC measurements demonstrated that the specimens were never pure tetragonal or monoclinic crystals. There were always some inclusions of the other form, so the crystals were mixtures of both forms. It is well seen on the exemplary DSC diagram of the first melting of the sample considered as monoclinic which is shown in Fig. 2. The recrystallization led always to a tetragonal form whose melting temperature is lower by about 4 degrees. It is shown in Fig. 2 as the second melting curve of the studied sample. The careful studies on numerous specimens allowed us to find that the melting temperature of the monoclinic form is equal to $390 \mathrm{~K}$ while the tetragonal form melts at $386 \mathrm{~K}$. The enthalpies of melting amount to about $23.8 \mathrm{~J} / \mathrm{mol}$ for both forms. Such a behaviour vs. temperature implies that the polymorphism of PCP is of the monotropic type. It means that the 


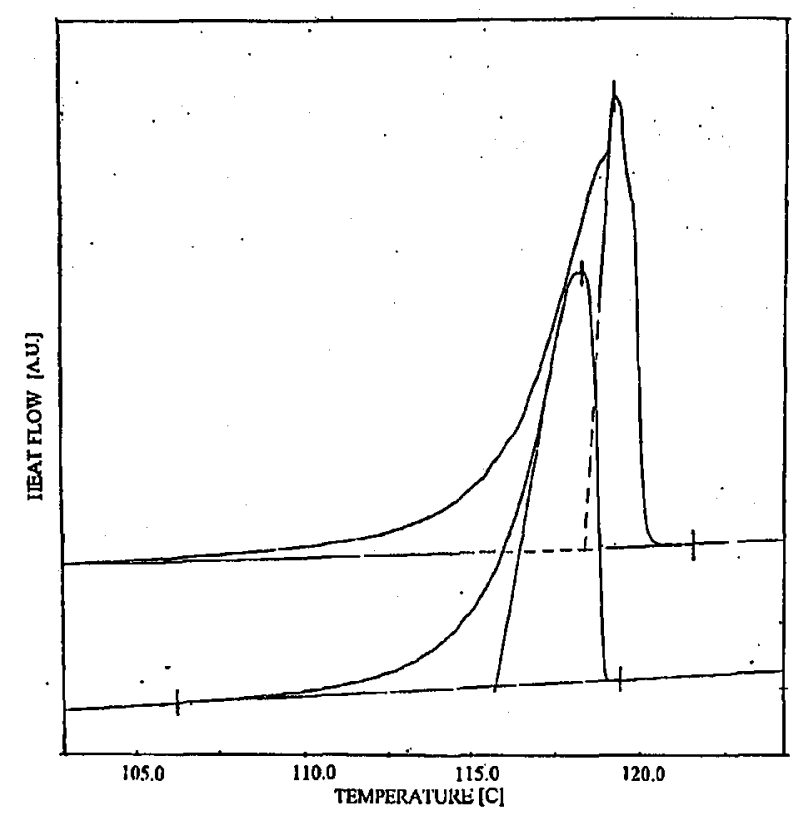

Fig. 2. The DSC diagram of the monoclinic PCP crystal. Upper curve - first heating cycle and bottom curve - second heating cycle.

monoclinic form is thermodynamically stable in the whole temperature range up to the melting point at $390 \mathrm{~K}$. The temperature of the equilibrium phase transition from the low-temperature monoclinic form to the high-temperature tetragonal form lies above the melting temperatures of both forms. The high-temperature tetragonal form crystallizes from melt and is metastable in the whole temperature range. These results corroborate the structural results [1] and the crystal energy calculations [2].

\section{The low-frequency IR and Raman spectra}

On the basis of group theory calculations one expects apart from 27 internal vibrations also $9(5 A+4 B)$ lattice optical vibrations which are allowed in the monoclinic crystal spectra and $21\left(2 A_{1}+A_{2}+2 B_{1}+2 B_{2}+7 E\right)$ lattice optical vibrations allowed in the tetragonal crystal spectra. All optical modes have mixed translational and librational character. Figure 3 shows the polarized far IR spectra of the tetragonal and monoclinic single crystal samples. They are very similar which reflects the similarity of molecular positions on the $(a, b)$ planes of both structures. The differences should be expected between the spectra polarized along the $c$ axis. Unfortunately we had no proper samples to be measured along this direction. The polarized low-frequency Raman spectra of the tetragonal PCP form are shown in Fig. 4. Their numerous bands are strongly depolarized i.e. are present in all polarizations differing only in intensity. The same holds for the monoclinic crystal. The twinning of the monoclinic crystals and the racemization of the tetragonal ones lie probably at the origin of that. The observed frequencies of the bands and 


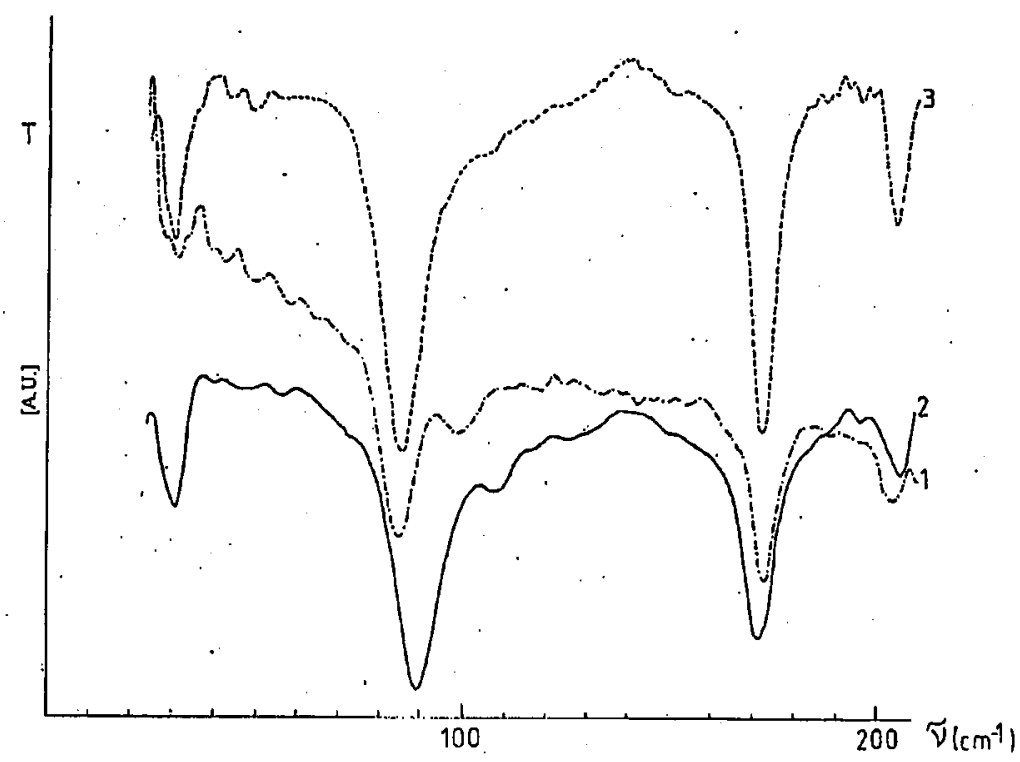

Fig. 3. The polarized far IR spectra of the monoclinic $(1-E\|a, 2-E\| b)$ and tetragonal $(3-E \| a)$ monocrystalline $\mathrm{PCP}$ at the ambient temperature.

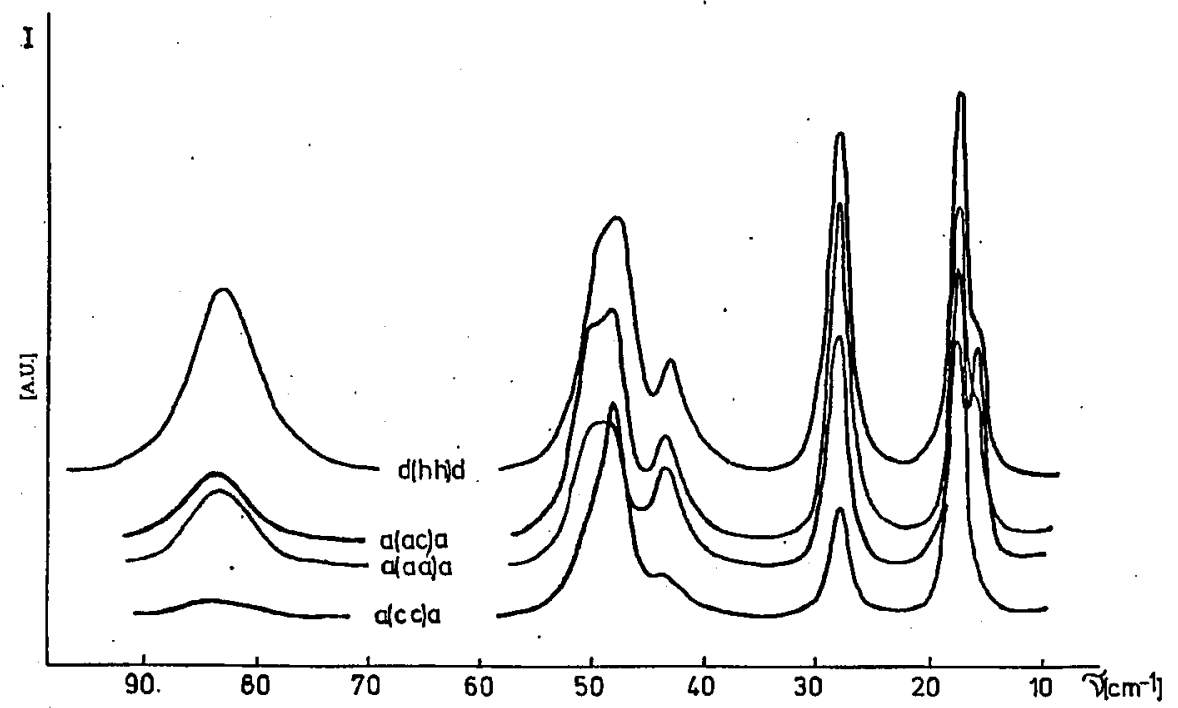

Fig. 4. The polarized Raman spectra of tetragonal monocrystalline PCP at the ambient temperature. The $(h, h)$ polarization is along the [110] direction.

their assignments are shown in Table II. Among them there are all low-frequency internal vibrations found by [4] in the IR spectra of powdered PCP and assigned as out-of-plane $\left(a_{2}, b_{2}\right)$ and in-plane $\left(a_{1}, b_{1}\right)$ ring modes. 
TABLE II

Frequencies $\left(\mathrm{cm}^{-1}\right)$, intensities, symmetries, polarizations and proposed assignments of bands in the low- frequency spectra of two polymorphs of PCP crystal at $190 \mathrm{~K}$ (tetragonal) and $87 \mathrm{~K}$ (monoclinic). The values in parentheses correspond to room temperature ${ }^{a}$.

\begin{tabular}{|c|c|c|c|c|c|}
\hline \multicolumn{2}{|c|}{ Tetragonal } & \multicolumn{3}{|c|}{ Monoclinic } & \multirow{3}{*}{$\begin{array}{l}\text { Proposed } \\
\text { assign- } \\
\text { ment } \\
\text { (int. vib. } \\
\text { acc. to [4]) }\end{array}$} \\
\hline \multirow[t]{2}{*}{ Raman } & \multirow{2}{*}{$\begin{array}{c}\text { IR } \\
E(E \| a)\end{array}$} & \multirow[t]{2}{*}{ Raman } & \multicolumn{2}{|c|}{ IR } & \\
\hline & & & $\overline{A(E \| b)}$ & $\overline{B(E \| a)}$ & \\
\hline $17.5 \mathrm{~s}(15)$ & & & & & lattice \\
\hline $18.5 \mathrm{~s}(17)$ & & & & & lattice \\
\hline $29 \mathrm{~s}(28)$ & & & & & lattice \\
\hline & $33 \mathrm{~m}(31)$ & & & & $\mathrm{T}^{b}$ \\
\hline & & & & $34.5 \mathrm{~m}(31)$ & $\mathrm{T}^{b}$ \\
\hline & & & $35.5 \mathrm{~m} \mathrm{(31)}$ & & $\mathrm{T}^{b}$ \\
\hline & & $41.5 \mathrm{~s}(36.5)$ & & & lattice \\
\hline $46.5 \mathrm{~s}(43.5)$ & & 47s (41.5) & & & lattice \\
\hline $51.5 \mathrm{~s}(48)$ & & $51 \mathrm{~s}(46.5)$ & & & lattice \\
\hline & & $57.5 \mathrm{vw}(51.5)$ & & & lattice \\
\hline $54 \mathrm{~s}(51)$ & & $59.5 \mathrm{~s}(51.5)$ & & & lattice \\
\hline & $75 \mathrm{sh}$ & & $75 \mathrm{sh}$ & $75 \mathrm{sh}$ & $?$ \\
\hline & $85 w(85,5)$ & & & & $?$ \\
\hline $86 \mathrm{~s}(83.5)$ & $88 \mathrm{~s}(85.5)$ & $89 \mathrm{~s}(84.5)$ & & $89 s(84.5)$ & $a_{2}$ \\
\hline & & $90.5 w(84.5)$ & & & \\
\hline & & $95.5 \mathrm{~m}(84.5)$ & $95 s(89)$ & & $b_{2}$ \\
\hline & $105 w(97)$ & & $102 w(96)$ & $102 \mathrm{~m}(98)$ & lattice \\
\hline & & $110 w$ & $110 w(105)$ & & lattice \\
\hline & $173.5 \mathrm{~s}(171)$ & & $176 \mathrm{~s}(171)$ & $178 \mathrm{~s}(172)$ & $b_{2}$ \\
\hline & & & $179 w(171)$ & & \\
\hline & $204 \mathrm{~m}$ & & & $203 \mathrm{~m}$ & $a_{1}$ \\
\hline & & & $205 \mathrm{~m}$ & & $b_{1}$ \\
\hline
\end{tabular}

${ }^{a} \mathrm{~s}=$ strong, $\mathrm{m}=$ medium, $\mathrm{w}=$ weak, $\mathrm{v}=$ very, $\mathrm{sh}=$ shoulder.

${ }^{b} \mathrm{~T}$ - translational mode.

The far-IR spectra are also very similar to the spectra of 2,6-dichloropyridine and the high-temperature phase of 3,5-dichloropyridine in spite of the different crystal symmetries of the compounds which belong to the centrosymmetrical $P 2_{1} / m$ space group [5]. It seems that the centre of symmetry in the non-centrosymmetrical structures of the two forms of PCP can be realized on the $(a, b)$ plane owing to the twinning of the monoclinic crystals and the racemic forms of the tetragonal ones. The mutual exclusion rule which seems to be fulfilled in the far IR and Raman spectra of both forms of PCP supports the statement. 
It is interesting that the lattice vibrations at very low frequencies (17.5, 18.5 and $29 \mathrm{~cm}^{-1}$ ) have their bands only in the Raman spectra of the tetragonal phase. It may be understood in terms of doubling of the $c$ lattice constant in the tetragonal unit cell of PCP with respect to the monoclinic one. Evidently the dispersion curves in the first Brillouin zone have a considerable slope. When the zone "folds" passing from the monoclinic $c^{*}$ constance to the tetragonal one the additional frequencies occur for $q=0$ value in the tetragonal crystal.

\section{The evidence of the monotropic phase transition}

In case of monotropy the equilibrium transition between two phases is situated at the temperature higher than the melting points and cannot be observed during heating or cooling cycles. Nevertheless, the high-temperature phase is metastable and in favourable conditions it may transform into the stable phase during a relaxation process. An activation energy is needed to pass over an energy barrier. These transitions are called monotropic and are frequently observed in liquid crystals [6]. We observed such a transformation while measuring the Raman spectra of the tetragonal crystal on cooling (Fig. 5). The phase transition took place between $190 \mathrm{~K}$ and $184.5 \mathrm{~K}$.

Also the observations of interference figures under crossed polarizers allowed us to see the tetragonal $\rightarrow$ monoclinic transition (Fig. 6). The interference figures of the two forms are distinctly different owing to their different crystal symmetries: the tetragonal form is optically uniaxial while the monoclinic one is biaxial. The transformation observed during cooling occurred abruptly at temperatures from $185 \mathrm{~K}$ to $170 \mathrm{~K}$ depending on rate of cooling and probably the defects in the crystal structure. Such a behaviour indicates the non-equilibrium character of the phase transition.

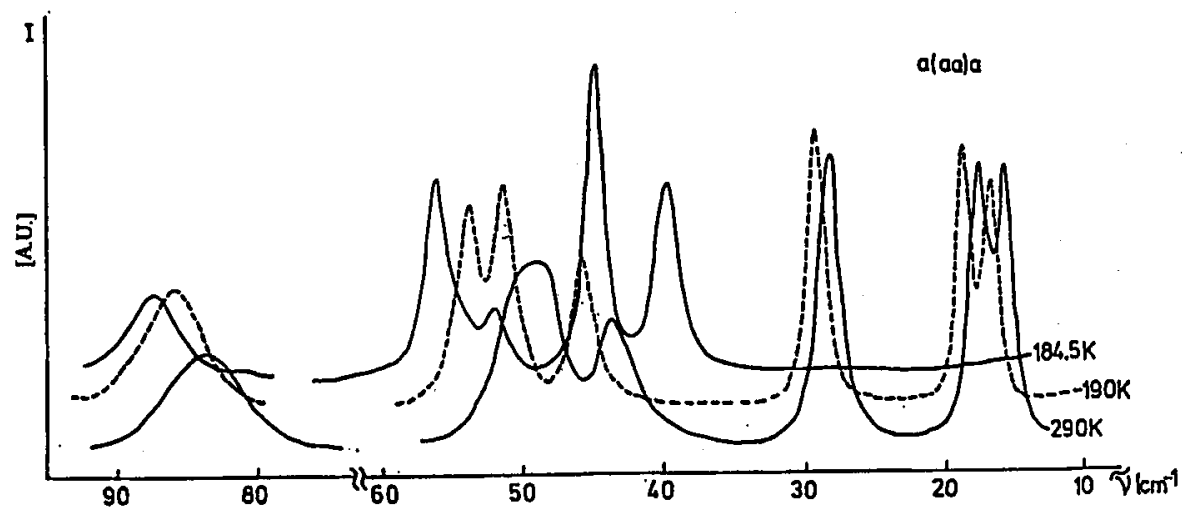

Fig. 5. The polarized Raman spectra of tetragonal PCP measured as a function of temperature. 

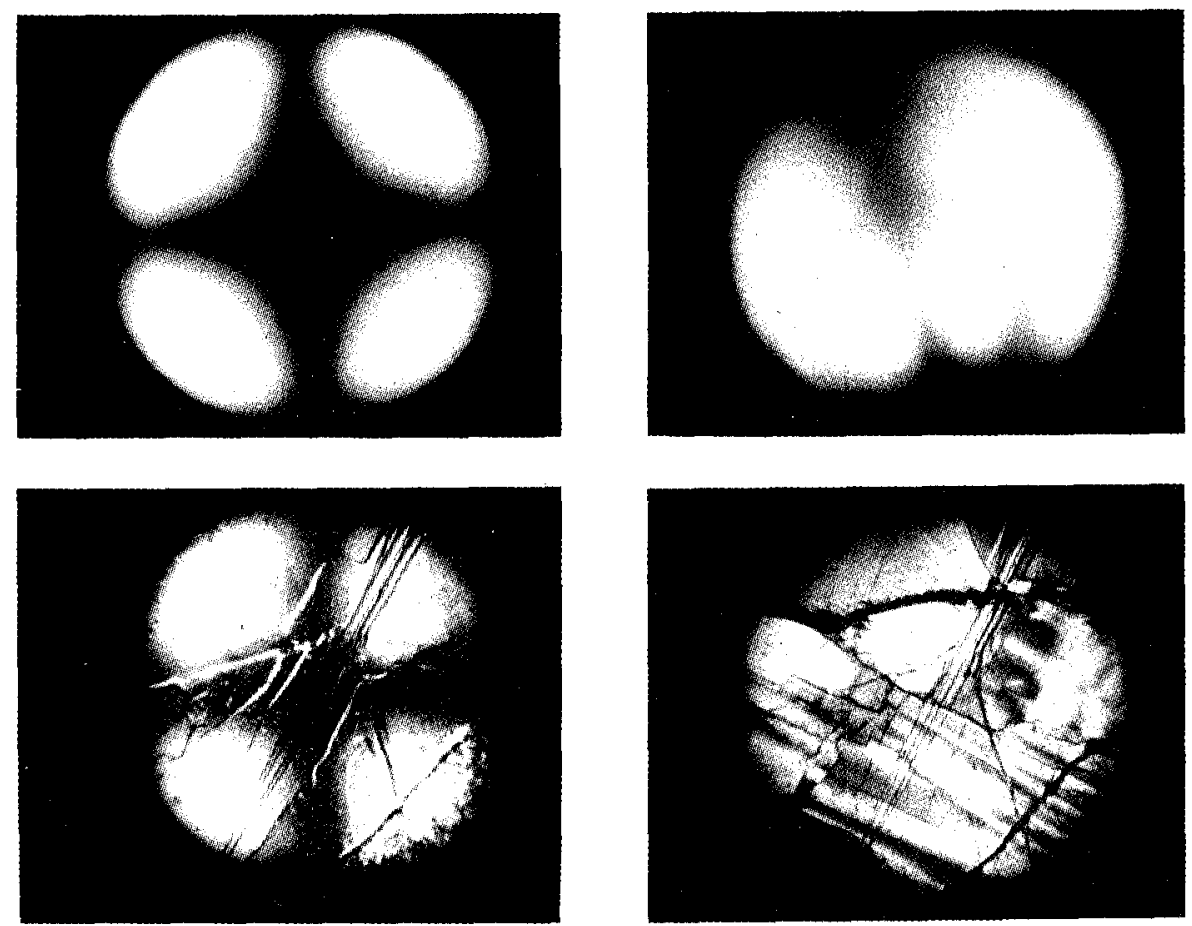

Fig. 6. The interference figures on the (001) plane of the tetragonal (left) and monoclinic (right) PCP crystal measured at room temperature (top) and before and after the transformation to the monoclinic phase (bottom) at about $185 \mathrm{~K}$.

At first the DSC studies gave no evidence of any anomaly in the whole temperature range from liquid nitrogen temperature up to the melting point which was consistent with the reports of Ref. [1]. Finally, we found a very weak anomaly at $193 \mathrm{~K}$ during the cooling cycle of the tetragonal phase sample. Its shape corresponds to the typical anomaly in case of the non-equilibrium transition of an over-cooled solid [7].

\section{Summary}

The polymorphic modifications of PCP are energetically almost equivalent and for that reason they grow simultaneously from solution or during sublimation. The twinned monoclinic crystals can imitate the tetragonal structure and intergrow with the tetragonal crystals [1]. As concerns the tetragonal phase it easily forms the racemate mixtures. The phenomena result in frequent growing of disordered crystals which happen to be the mixed crystals of the D- and L-forms of the tetragonal polymorph and the monoclinic one. Therefore the $\alpha$ angle characterising the optical activity of the tetragonal crystal ranges from $-20^{\circ} / \mathrm{mm}$ to $+20^{\circ} / \mathrm{mm}$ depending on a specimen [1]. Finally, the two polymorphic modifications are difficult to separate. 
The polymorphs are monotropic forms, their melting temperatures differ by about 4 degrees. The tetragonal form is metastable in the whole temperature range up to the melting point. It may transform into the stable, monoclinic phase during a non-equilibrium monotropic transformation.

\section{References}

[1] H.J. Russel, H.G. Scott, J. Cryst. Mol. Struct. 3, 259 (1973).

[2] A. Gavezzotti, M. Simonetta, Acta Crystallogr. A 31, 645 (1975).

[3] W.J. Kusto, J. Meinnel, Phase Transit. 35, 105 (1991).

[4] R.T. Bailey, G.P. Strachan, Spectrochim. Acta A 26, 1129 (1970).

[5] F. Romain, P. Tougard, B. Pasquier, N. Le Calve, A. Novak, A. Peneau, L. Guibe, J. Ramakrishna, Phase Transit. 3, 259 (1983).

[6] M. Rachwalska, Mol. Cryst. Liq. Cryst. 237, 151 (1993).

[7] D. Fox, Physics and Chemistry of Organic Solid State, Wiley, New York 1963. 